\title{
Maintenance of improved lipid levels following attendance at a cardiovascular risk reduction clinic: a I0-year experience
}

\author{
Glen J Pearson ${ }^{1,5}$ \\ Kari L Olson ${ }^{6}$ \\ Nicole E Panich' \\ Sumit R Majumdar 2,5 \\ Ross T Tsuyuki ${ }^{1,4}$ \\ Dawna M Gilchrist ${ }^{2,5}$ \\ Ali Damani ${ }^{4}$ \\ Gordon A Francis ${ }^{3,5}$ \\ The MILLARR Study (Maintenance \\ of Improved Lipid Levels Following \\ Attendance at a Cardiovascular Risk \\ Reduction Clinic) \\ 'Department of Medicine, Divisions \\ of Cardiology; ${ }^{2}$ General Internal \\ Medicine; ${ }^{3}$ Endocrinology and \\ Metabolism; Faculty of Pharmacy and \\ Pharmaceutical Sciences; ${ }^{4}$ University \\ of Alberta, Edmonton, Alberta, Canada; \\ ${ }^{5}$ Cardiovascular Risk Reduction \\ Clinic (CRRC), University of Alberta \\ Hospital, Edmonton, Alberta, Canada; \\ 'University of Colorado Health \\ Sciences Center, Denver, Colorado, \\ USA; ${ }^{7}$ Family Medicine (Private \\ Practice), Calgary, Alberta, Canada
}

Correspondence: Glen J Pearson Division of Cardiology, University of Alberta; Suite 2EI.24, Walter C. Mackenzie Health Sciences Centre; 8440-I I2th Street; Edmonton, Alberta, Canada;T6G 2B7

Tel + I 780407 I590

Fax +I 780407 I496

Email glen.pearson@ualberta.ca
Background: Specialty cardiovascular risk reduction clinics (CRRC) increase the proportion of patients attaining recommended lipid targets; however, it is not known if the benefits are sustained after discharge. We evaluated the impact of a CRRC on lipid levels and assessed the long-term effect of a CRRC in maintaining improved lipid levels following discharge.

Methods: The medical records of consecutive dyslipidemic patients discharged $>6$ months from a tertiary hospital CRRC from January 1991 to January 2001 were retrospectively reviewed. The primary outcome was the change in patients' lipid levels between the final CRRC visit and the most recent primary care follow-up. A worst-case analysis was conducted to evaluate the potential impact of the patients in whom the follow-up lipid profiles post-discharge from the CRRC were not obtained.

Results: Within the CRRC (median follow-up $=1.28$ years in 1064 patients), we observed statistically significant improvements in all lipid parameters. In the 411 patients for whom post-discharge lipid profiles were available (median follow-up $=2.41$ years), there were no significant differences observed in low-density lipoprotein-cholesterol, total cholesterol (TC), or triglycerides since CRRC discharge; however, there were small improvements in high-density lipoprotein-cholesterol (HDL-C) and TC:HDL ratio ( $p<0.05$ for both). The unadjusted worst-case analysis (653 patients with no follow-up lipid profiles) demonstrated statistically significant worsening of all lipid parameters between CRRC discharge and the most recent follow-up. However, when the change in lipid parameters between the baseline and the most recent follow-up was assessed in this analysis, the changes in all lipid parameters were significantly improved $(\mathrm{p}<0.05)$.

Conclusions: This study demonstrates that a CRRC can improve lipid levels and suggests that these benefits are sustained once patients are returned to the care of their primary physician.

Keywords: cardiovascular risk factors, dyslipidemia, outcomes, pharmacotherapy, secondary prevention

\section{Introduction}

Cardiovascular disease (CVD) is the leading cause of death among adults worldwide (Murray and Lopez 1997), and in Canada it accounts for $37 \%$ of total mortality (Heart and Stroke Foundation of Canada 1999; Fodor et al 2000). Dyslipidemias, in particular elevated low-density lipoprotein-cholesterol (LDL-C) or decreased high-density lipoprotein cholesterol (HDL-C), are important risk factors for the development of vascular disease and recognized to be major independent risk factors for coronary heart disease (CHD) (Castelli et al 1986; Anderson et al 1987; Neaton et al 1992; Stamler et al 1996). Evidence from several large randomized controlled trials have demonstrated the benefit of treating patients both with and without documented CVD across the spectrum of cholesterol levels with various lipid-lowering medications. A meta-analysis of these trials demonstrated that intervention with hydroxymethyl 
glutaryl-coenzyme A reductase inhibitors (statins) showed a large and statistically significant reduction in mortality from CHD (19\% per mmol/L reduction in LDL-C) and from all-causes $(12 \%$ per $\mathrm{mmol} / \mathrm{L}$ reduction in $\mathrm{LDL}-\mathrm{C})$ (Baigent et al 2005).

On the basis of this robust cumulative evidence, expert groups have promulgated and regularly updated national guidelines to assist health care practitioners with the treatment of patients with dyslipidemias (NCEP ATP III 2002; $\mathrm{McPherson}$ et al 2006). The latest updates of these guidelines provide a strategy to risk stratify patients according to data adapted from the Framingham study, based on their individual estimated 10-year risk of experiencing a cardiac endpoint such as a nonfatal myocardial infarction or death from CHD (Grundy et al 1999; NCEP ATP III 2002; McPherson et al 2006). Despite these efforts, numerous practice-pattern analyses indicate that the treatment of patients is suboptimal. Patients are under-diagnosed, under-treated and are not achieving lipoprotein targets (Schrott et al 1997; Majumdar et al 1999; Pearson et al 2000; Garcia et al 2004; Cooke and Hammerash 2006; Stacy and Egger 2006).

Lipid and cardiovascular risk reduction clinics (CRRC) have been developed to provide assistance in the management of patients with dyslipidemias. A number of studies have evaluated the effectiveness of these specialty clinics. Compared to management by primary care physicians or specialists in other nonlipid clinics, patients who are managed in CRRC or lipid clinics have lower LDL-C levels and are more likely to achieve their lipid targets (Shaffer and Wexler 1995; Harris et al 1998; Wilson et al 1999; Yates et al 2001; Gavish et al 2002; Koren and Hunninghake 2004; Olson et al 2005). Additional evidence demonstrates that patients referred to a CRRC were also able to achieve significant improvements in other cardiovascular $(\mathrm{CV})$ risk factors, such as blood pressure and glycemic control (Olson et al 2005).

While previous studies have evaluated the effectiveness of reducing lipid levels within the clinic itself or in comparison to primary care practices, to our knowledge no study has determined the level of lipid control for patients subsequent to their discharge from these clinics. This is important since there is uncertainty as to whether patients should be discharged, at some point in their care, back to their primary care physician or continued to be followed in the CRRC. Clearly, this has significant resource implications for these clinics and their patients. The purpose of this study was to determine the long-term impact on lipid control among patients who were initially managed in a CRRC and subsequently discharged to primary care follow-up.

\section{Methods}

\section{Study design}

This was a retrospective cohort study among consecutive patients followed within a single CRRC at the University of Alberta Hospital in Edmonton, Alberta, Canada. The Canadian health care system provides for universal access to essential medical and physician services. Prescription drug coverage is provided provincially for seniors (age $\geq 65$ years) and the indigent; otherwise, third party drug insurance is paid for by individuals and/or their employers. This clinic utilizes a multi-factorial risk reduction model, which targets individualized changes in lifestyle and pharmacologic treatment to decrease patients' risk of developing or worsening CVD. The clinic is staffed $1 / 2$ to 1 day per week by a dedicated multidisciplinary team of physicians (2 to 3 per clinic day), pharmacists, nurses, and dieticians. Together, the clinic team provides a comprehensive program that consists of a combination of education and counseling (nutrition, smoking cessation, and physical activity), behavioral interventions and pharmacotherapy, individually tailored to each patient to optimally reduce their $\mathrm{CV}$ risk profile. In addition to inperson follow-up for patients within the clinic, the CRRC also provides intermittent telephone follow-up for patients when necessary.

The study was reviewed and approved by the Health Research Ethics Board at the University of Alberta.

\section{Patients}

Our study group was comprised of consecutive patients referred to the CRRC for either primary or secondary prevention of CVD between January 1991 and January 2001. Patients were identified from clinic records and determined to be eligible for this study if they were: $\geq 18$ years of age, had any form of dyslipidemia (elevation of any component of the fasting lipid profile) at the time of referral, attended a minimum of one clinic visit, and had at least one follow-up lipid profile reflecting CRRC management, and had been discharged from the CRRC back to their primary care physician for at least 6 months. Patients were excluded from the study if their primary care physician refused to participate or failed to respond to the investigators' repeated requests for study related data or the primary care physician did not have a follow-up lipid profile for the patient at least 6 months after they were discharged from the CRRC.

\section{Procedures}

The primary care or referring physician for each patient fulfilling the eligibility criteria who was discharged from the 
CRRC was contacted to obtain the results of the most recent lipid profile. Participating physicians' offices were requested to fax a copy of their patients' most recent lipid profile report to the project office. In an attempt to optimize the number of patients included in the study, follow-up letter reminders to nonresponding physicians occurred in accordance to published criteria (Salant and Dillman 1994).

The medical records of all eligible patients were reviewed. The following data were abstracted: patient demographics (age, sex), complete fasting lipid profiles (total cholesterol [TC], LDL-C, HDL-C, triglycerides [TG], and TC:HDL-C ratio), and the number and nature of cardiac risk factors. Cardiac risk factors were defined as chart documentation by a physician of the following: hypertension, diabetes mellitus, smoking, previous myocardial infarction, percutaneous coronary intervention (PCI), coronary artery bypass graft surgery (CABG), angina, peripheral arterial disease (PAD) (ie, endarterectomy or symptoms suggestive of PAD), and cerebral vascular disease (transient ischemic attack or stroke). Additional cardiac risk information was collected, if available, including the presence of electrocardiogram documented left ventricular hypertrophy (LVH), impaired fasting glucose or impaired glucose tolerance, and family history of premature $\mathrm{CV}$ disease (history of CAD in a first-degree relative before the age of 55 years in a male or 65 years in a female). Lipidlowering medications prescribed during CRRC assessment and follow-up were also collected.

\section{Outcome measures and statistical analysis}

The primary outcome was the change in patients' lipid levels between the final CRRC visit (discharge) and the most recent lipid levels obtained by the patients' physician at least 6 months after they were discharged from the CRRC (follow-up). The secondary outcome of interest was the change in patients' lipid levels between the initial referral (baseline) and the final visit to the CRRC (discharge). The before and after changes in the lipid parameters (TC, LDL, HDL, TG, and TC:HDL) were compared using generalized least squares approach. This was employed due to missing data and to account for the assumption of unequal variance-covariance over time. The least square means and $95 \%$ confidence interval of the differences of means with Tukey-Kramer adjustments for pair-wise comparisons were provided for each interval change in the lipid parameters. The descriptive demographic characteristics of all patients and those patients remaining in the follow-up period after final visit to the CRRC were summarized using means for continuous variables and proportions for categorical variables. In order to evaluate the potential impact of the patients in whom the follow-up lipid profiles post-discharge from the CRRC were not obtained, a second analysis was conducted. The worst-case scenario was used for all patients without follow-up labs and their original baseline lipid values at the time of referral were imputed for the post-discharge follow-up values. Statistical significance was set at a p-value of $<0.05$. All statistical analyses were performed using SAS statistical software (version 8.2; SAS Institute Inc. Cary, NC).

\section{Results}

Figure 1 summarizes the overall disposition of patients in the study. There were 1,975 patients referred to the CRRC between January 1991 and January 2001. From this population, 1,064 patients (53.9\%) fulfilled the study eligibility criteria and were evaluated within the CRRC. The reasons for excluding 911 patients were: age less than 18 years $(\mathrm{n}=42)$, no diagnosis of a dyslipidemia at the time of referral $(\mathrm{n}=139)$, no follow-up lipid profile reflecting the CRRC management $(\mathrm{n}=269)$, not discharged from the CRRC at the time of the study or discharged for less than 6 months $(n=415)$, deceased $(n=22)$, and medical record unavailable for review $(n=24)$. Follow-up fasting lipid profiles were obtained for 411 (38.6\%) of the patients who were eligible for the study. Patient demographics for those with and without follow-up lipid profiles are outlined in Table 1 .

\section{Changes in lipid levels within the CRRC}

Overall, the impact of the CRRC management was assessed in 1064 patients (mean age $=51.9 \pm 12.4$ years; $41.8 \%$ female $)$ followed for a median duration of 1.28 years (mean number of clinic visits $=3.6$; mean additional follow-up contacts by telephone $=4.1)$. There were $473(44.5 \%)$ high-risk patients, with a documented history of vascular disease $(n=311)$ or diabetes and age $>30$ years $(n=162)$; the median CRRC follow-up in this sub-group was 1.57 years (mean number of clinic visits $=4.5$; mean additional follow-up telephone contacts $=5.8)$. The remaining $591(55.5 \%)$ patients were identified to have a mean of 2 cardiovascular risk factors. The distribution of risk factors, documented incidence of specific vascular disease, and other demographics are outlined in Table 1.

The CRRC achieved improvements in all lipid parameters (Table 2). The observed changes between the time of referral and discharge from the CRRC were decreases in LDL-C (19.1\%), TC (17.9\%), TG (40.3\%), and TC:HDL (22.8\%), 


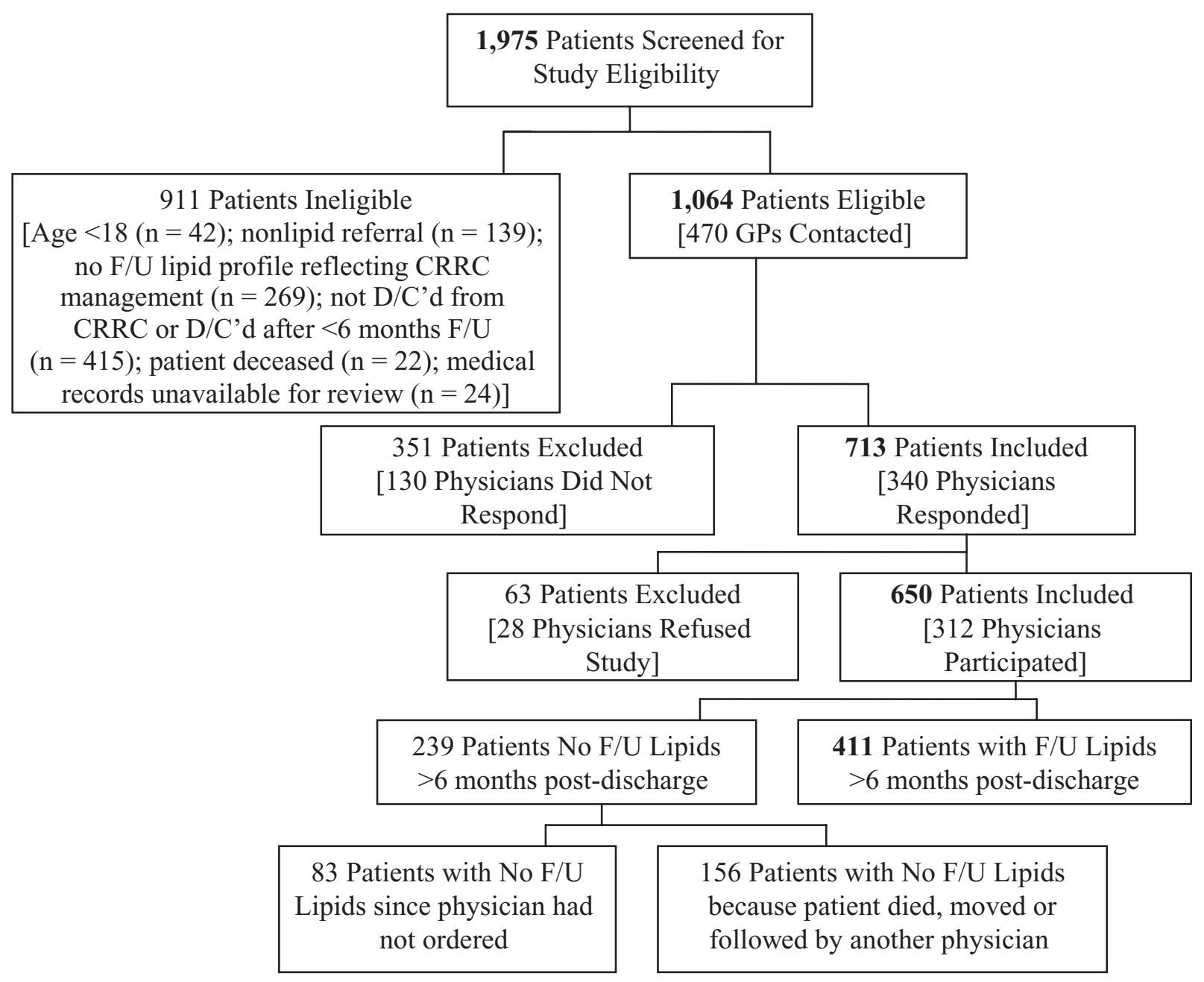

Figure I Flow diagram of study patient disposition.

Abbreviations:

Table I Patient demographics

\begin{tabular}{|c|c|c|c|}
\hline Parameter & All patients $(n=1064)$ & Patients with follow-up $(n=4 I I)$ & Patients with no follow-up $(n=653)$ \\
\hline Age (mean $\pm S D)$ & $51.9 \pm 12.4$ & $52.6 \pm 11.5$ & $57.4 \pm 13.0$ \\
\hline \multicolumn{4}{|l|}{ Sex } \\
\hline Males & $619(58.2 \%)$ & $215(52.3 \%)$ & $404(61.9 \%)$ \\
\hline Females & $445(41.8 \%)$ & $196(47.7 \%)$ & $249(38.1 \%)$ \\
\hline \multicolumn{4}{|l|}{ Documented vascular disease* } \\
\hline Prior myocardial infarction & $210(19.7 \%)$ & $75(18.2 \%)$ & 135 (20.7\%) \\
\hline Angina & 175 (16.4\%) & $62(15.1 \%)$ & $113(17.3 \%)$ \\
\hline Percutaneous coronary intervention & III (10.4\%) & $44(10.7 \%)$ & $67(10.3 \%)$ \\
\hline Coronary artery bypass graft surgery & $118(11.1 \%)$ & $43(10.5 \%)$ & 75 (1 I.5\%) \\
\hline Transient ischemic attack & $31(2.9 \%)$ & $12(2.9 \%)$ & $19(2.9 \%)$ \\
\hline Stroke & $32(3.0 \%)$ & $9(2.2 \%)$ & $23(3.5 \%)$ \\
\hline Peripheral vascular disease & $47(4.4 \%)$ & II (2.7\%) & $36(5.5 \%)$ \\
\hline Diabetes mellitus & $162(15.2 \%)$ & $63(15.3 \%)$ & $99(15.2 \%)$ \\
\hline $\begin{array}{l}\text { High risk patients (history of vascular } \\
\text { disease or diabetes) }\end{array}$ & $473(44.4 \%)$ & $172(4 \mid .8 \%)$ & $30 I(46.1 \%)$ \\
\hline
\end{tabular}


Table I. (continued)

\begin{tabular}{|c|c|c|c|}
\hline Parameter & All patients $(n=1064)$ & Patients with follow-up $(n=4 \mathrm{I} I)$ & Patients with no follow-up $(n=653)$ \\
\hline \multicolumn{4}{|l|}{ Distribution of risk factors } \\
\hline Hypertension & $390(36.7 \%)$ & I5I (36.7\%) & $239(36.6 \%)$ \\
\hline Current smoker & $214(20.1 \%)$ & $80(19.5 \%)$ & $134(20.5 \%)$ \\
\hline Positive family history & $642(60.3 \%)$ & $251(61.1 \%)$ & $391(59.9 \%)$ \\
\hline Left ventricular hypertrophy & $29(2.7 \%)$ & $5(1.2 \%)$ & $24(3.7 \%)$ \\
\hline \multicolumn{4}{|l|}{ Other factors } \\
\hline $\begin{array}{l}\text { Impaired fasting glucose or impaired } \\
\text { glucose tolerance }\end{array}$ & $69(6.5 \%)$ & $27(6.6 \%)$ & $42(6.4 \%)$ \\
\hline Solid organ transplant & 47 (4.4\%) & $8(1.9 \%)$ & $39(6.0 \%)$ \\
\hline $\begin{array}{l}\text { Number of risk factors identified } \\
\text { among nonhigh risk patients (mean) }\end{array}$ & 2 & 2 & 2 \\
\hline
\end{tabular}

Note: *not mutually exclusive.

as well as an increase in HDL-C (8.9\%), which were all statistically significant $(p<0.0001)$. These changes were achieved with a high frequency of lipid-lowering medication use (Table 3). There were a total of 1,593 lipid-lowering medication prescriptions in the 1,064 patients (average of 1.5 medications/patient) used during CRRC follow-up. Statins were the most commonly used class of agents, with $82.7 \%$ of patients being prescribed one of these agents while in the CRRC. Fibrates were prescribed in $37.9 \%$ of patients, niacin in $13 \%$ and a resin in $6.1 \%$. In addition, combination lipid-lowering regimens (Table 4) were utilized with a high frequency in this population, with $19.1 \%$ of patients being prescribed combination therapy at some point in time; a twodrug combination regimen was used in $18.6 \%$ of patients and $0.5 \%$ of patients used three-drug combination therapy. The most frequently prescribed combination regimen consisted of a statin plus a fibrate, which was used in $13.1 \%$ of patients.

\section{Post-discharge from the CRRC}

To assess the impact of lipid control after patients were discharged from the CRRC, complete follow-up lipid profiles were assessed in 411 patients. The mean age of this group was $52.6 \pm 11.5$ years; $47.7 \%$ were females and $172(41.8 \%)$ were high-risk patients. The median duration between CRRC discharge and the most recent lipid profile was 2.41 years. Compared to the total population evaluated within the CRRC, there were no obvious differences in these patients with regard to the incidence of documented vascular disease, diabetes, or other risk factors for $\mathrm{CV}$ disease (Table 1). Table 5 outlines differences in fasting lipid profiles between CRRC discharge and the most recent follow-up post-discharge. There were no significant differences observed in LDL-C, TC, or TG. However, there were small, but statistically significant, improvements in HDL-C $(+0.03 \mathrm{mmol} / \mathrm{L} ; \mathrm{p}<0.05)$ and TC:HDL ratio $(-0.20 ; \mathrm{p}<0.05)$ observed in the most recent follow-up lipid profiles of these patients. Analysis of the highrisk sub-group demonstrated no significant change in any of the lipid parameters between discharge from the CRRC and the most recent follow-up.

In the worst-case analysis, the original baseline lipid values at the time of referral were imputed for the 653 patients for whom follow-up labs could not be obtained, and

Table 2 Change in mean lipid levels in I,064 patients during CRRC attendance

\begin{tabular}{lllll}
\hline Lipid parameter & Visit I -(Baseline) & Final visit (Discharge) & Change \\
\hline TC $(\mathrm{mmol} / \mathrm{L})$ & & & $\frac{\text { Mean (\%) }}{\text { p-value }}$ \\
LDL-C (mmol/L) & 6.99 & 5.73 & $-1.25(17.9)$ & $-0.81(19.1)$ \\
HDL-C (mmol/L) & 4.25 & 3.39 & $+0.10(8.9)$ & $<0.0001$ \\
TG $(\mathrm{mmol} / \mathrm{L})$ & 1.12 & 1.21 & $-1.81(40.3)$ & $<0.0001$ \\
TC:HDL & 4.49 & 2.74 & $-1.47(22.8)$ & $<0.0001$ \\
\hline
\end{tabular}

Notes: Median duration of follow-up in the CRRC (time interval between Clinic Visit I and Final Visit) $=1.28$ years. Abbreviation: CRRC, Cardiovascular Risk Reduction Clinic. 
Table 3 Frequency of lipid-lowering medications used (at any time) during CRRC attendance

\begin{tabular}{|c|c|c|c|}
\hline Medication & & Frequency (\# of instances used) & $\%$ Patients $(n=1064)$ \\
\hline \multirow[t]{7}{*}{ HMG-CoA (statin) } & & 880 & 82.7 \\
\hline & Atorvastatin & 260 & 24.4 \\
\hline & Pravastatin & 238 & 22.4 \\
\hline & Simvastatin & 236 & 22.2 \\
\hline & Lovastatin & 76 & 7.1 \\
\hline & Fluvastatin & 45 & 4.2 \\
\hline & Cerivastatin & 25 & 2.3 \\
\hline \multirow[t]{4}{*}{ Fibric acid derivative (fibrate) } & & 508 & 37.9 \\
\hline & Fenofibrate & 386 & 26.4 \\
\hline & Gemfibrozil & 102 & 9.6 \\
\hline & Bezafibrate & 20 & 1.9 \\
\hline \multirow[t]{3}{*}{ Nicotinic acid (niacin) } & & 138 & 13.0 \\
\hline & Regular-release & 104 & 9.8 \\
\hline & Timed-release & 34 & 3.2 \\
\hline \multirow[t]{3}{*}{ Bile acid sequestrants (resin) } & & 65 & 6.1 \\
\hline & Cholestyramine & 39 & 3.7 \\
\hline & Colestipol & 26 & 2.4 \\
\hline Others (Clofibrate, Salmon Oil) & & 2 & 0.2 \\
\hline
\end{tabular}

Abbreviations: CRRC, Cardiovascular Risk Reduction Clinic; HMG-CoA, 3-hydroxy-3-methyl-glutaryl-CoA reductase.

this data was combined with that for the 411 patients with follow-up lipid values. For 414 patients (39\%), no reply for follow-up lipid profile results was obtained from the original referring physician (351 patients; 33\%) or the referring physician refused to participate in the study (63 patients; $6 \%$ ). For 239 patients (22.5\%), the referring physician replied but was unable to provide the patient's recent lipid profile. Of these, recent lipid values were not available for 156 patients (14.7\%) because the physician no longer cared for the patient (died, moved, or switched physicians) and there were 83 patients (7.8\%) still under the referring physician's care in whom no recent follow-up lipid screen had been ordered. The 653 patients with no follow-up data were older and had a larger percentage of male patients compared to the group with follow-up data, but otherwise appeared to be similar to those patients for whom follow-up labs were available. The unadjusted worst-case analysis demonstrated a statistically significant worsening of all lipid parameters between CRRC discharge and the most recent follow-up (Table 6). However, when the change in lipid parameters between the

Table 4 Frequency of various combination regimens used (at any time) during CRRC attendance

\begin{tabular}{lll}
\hline Combination regimen & Frequency (\#of Instances Used) & \% Patients $(\mathbf{n}=\mathbf{~ I 0 6 4 )}$ \\
\hline Statin + Fibrate & 139 & $13.1 \%$ \\
Statin + Niacin & 25 & $2.4 \%$ \\
Statin + Resin & 22 & $2.1 \%$ \\
Statin + Fish Oil & 1 & $0.1 \%$ \\
Fibrate + Niacin & 7 & $0.7 \%$ \\
Fibrate + Resin & 2 & $0.2 \%$ \\
Niacin + Resin & 2 & $0.2 \%$ \\
Statin + Niacin + Fibrate & 3 & $0.3 \%$ \\
Statin + Niacin + Resin & I & $0.1 \%$ \\
Statin + Fibrate + Resin & 1 & $0.1 \%$ \\
TOTAL & 203 & $19.1 \%$ \\
\hline
\end{tabular}

Abbreviation: CRRC, Cardiovascular Risk Reduction Clinic. 
Table 5 Change in mean lipid levels in 4 I I patients following discharge from the CRRC

\begin{tabular}{|c|c|c|c|c|c|c|}
\hline \multirow[t]{2}{*}{ Lipid parameter } & \multirow{2}{*}{$\begin{array}{l}\text { Visit } \\
\text { I-(Baseline) }\end{array}$} & \multirow{2}{*}{$\begin{array}{l}\text { Final visit } \\
\text { (Discharge) }\end{array}$} & \multirow[t]{2}{*}{ Follow-up* } & \multicolumn{3}{|c|}{ Mean change $\left(95 \% \mathrm{Cl}^{\dagger}\right)$} \\
\hline & & & & Visit I to final visit & Visit I to follow-up & Discharge to Follow-up* \\
\hline $\mathrm{TC}(\mathrm{mmol} / \mathrm{L})$ & 7.13 & 5.81 & 5.77 & $-1.32^{\ddagger}(-1.59,-1.04)$ & $-1.36^{\ddagger}(-1.64,-1.07)$ & $-0.04(-0.19,+0.1)$ \\
\hline LDL-C (mmol/L) & 4.34 & 3.44 & 3.37 & $-0.9)^{\ddagger}(-1.07,-0.73)$ & $-0.97^{\ddagger}(-1.14,-0.80)$ & $-0.07(-0.21,+0.07)$ \\
\hline HDL-C (mmol/L) & 1.13 & 1.24 & 1.27 & $+0.1 I^{\ddagger}(+0.08,+0.14)$ & $+0.14^{\ddagger}(+0.11,+0.17)$ & $+0.03^{\ddagger}(+0.01,+0.06)$ \\
\hline TG (mmol/L) & 4.84 & 2.62 & 2.64 & $-2.22^{\ddagger}(-3.11,-1.34)$ & $-2.20^{\ddagger}(-3.11,-1.30)$ & $+0.02(-0.20,+0.24)$ \\
\hline TC:HDL & 6.54 & 5.05 & 4.85 & $-1.49 \ddagger(-1.71,-1.27)$ & $-1.69^{\ddagger}(-1.95,-1.42)$ & $-0.20^{\ddagger}(-0.39,0.006)$ \\
\hline
\end{tabular}

Notes: *Median duration of follow-up since CRRC discharge $=2.41$ years (minimum $=0.5$ years). ${ }^{\dagger}$ Tukey-Kramer adjustment. ${ }^{\ddagger} \mathrm{p}<0.05$.

Abbreviations: CRRC, Cardiovascular Risk Reduction Clinic; HDL-C, high-density lipoprotein-cholesterol; LDL-C, low-density lipoprotein-cholesterol;TC, total cholesterol; TG, triglycerides.

baseline (visit 1) and the most recent follow-up was assessed in this analysis, the reductions in TC $(-0.53 \mathrm{mmo} / \mathrm{L}), \mathrm{LDL}-\mathrm{C}$ $(-0.39 \mathrm{mmol} / \mathrm{L})$, TG $(-0.83 \mathrm{mmol} / \mathrm{L})$, and TC:HDL ratio $(-0.67)$ and the increase in HDL $(+0.06 \mathrm{mmol} / \mathrm{L})$ were all statistically significant $(\mathrm{p}<0.05)$.

\section{Discussion}

In an attempt to improve the management of dyslipidemias in patients with CHD or at high-risk for developing future CVD events, specialty clinics have been developed within many institutions. Studies evaluating the effectiveness of these specialty clinics have demonstrated significant improvements in lowering LDL-C levels, achieving lipid targets, and reducing other cardiac risk factors compared to usual care (Shaffer and Wexler 1995; Harris et al 1998; Wilson et al 1999; Yates et al 2001; Gavish et al 2002; Koren and Hunninghake 2004; Olson et al 2005). While the evidence clearly demonstrates that specialty cardiovascular risk reduction or lipid clinics are very effective in managing patients with dyslipidemias and other risks for CVD, all published evaluations of these practices have focused on the outcomes achieved while the patients are managed within the clinic. The present study demonstrated that the improvements achieved in all lipid parameters during attendance at the CRRC were maintained in both high-risk and moderate-risk patients over a median follow-up of 1.28 years. During this time patients were seen in the clinic for a mean of 3.6 visits and received an additional 4.1 clinic telephone contacts.

The very high frequency of medication use by the CRRC is one probable explanation for the significant change in lipids observed in the patients during clinic follow-up. An average of 1.5 lipid-lowering medications/patient used during CRRC follow-up reflects a high rate of medication change for efficacy or toxicity reasons, as well as the need for combination therapy in many patients (19.1\%). In a previous study within our population, we demonstrated that commonly prescribed combination regimens (statin + fibrate and statin + niacin) were safe and effective when patients are well informed about the potential toxicities and judiciously monitored (Taher et al 2002). Not surprisingly, statins were the class of lipid-lowering agents most commonly prescribed, used in $>82 \%$ of our patients. In addition to

Table 6 Change in mean lipid levels in patients $(n=1064)$ following discharge from the CRRC by worst-case analysis

\begin{tabular}{|c|c|c|c|c|c|}
\hline \multirow[t]{2}{*}{ Lipid parameter } & \multirow[t]{2}{*}{ Visit I-(Baseline) } & \multirow[t]{2}{*}{ Final visit (Discharge) } & \multirow[t]{2}{*}{ Follow-up } & \multicolumn{2}{|c|}{ Mean** change $\left(95 \% \mathrm{Cl}^{\dagger}\right)$} \\
\hline & & & & Visit I to follow-up & Discharge to follow-up \\
\hline $\mathrm{TC}(\mathrm{mmol} / \mathrm{L})$ & 6.98 & 5.73 & 6.46 & $-0.53^{\ddagger}(-0.63,-0.43)$ & $+0.72^{\ddagger}(+0.58,+0.86)$ \\
\hline LDL-C (mmol/L) & 4.1 & 3.38 & 3.75 & $-0.39 \ddagger(-0.46,-0.33)$ & $+0.38^{\ddagger}(+0.29,+0.46)$ \\
\hline HDL-C (mmol/L) & 1.11 & 1.21 & 1.17 & $+0.06^{\ddagger}(+0.04,+0.07)$ & $-0.04^{\ddagger}(-0.06,-0.02)$ \\
\hline TG (mmol/L) & 4.53 & 2.73 & 3.70 & $-0.83^{\ddagger}(-1.12,-0.54)$ & $+0.97^{\ddagger}(+0.68,+1.25)$ \\
\hline TC:HDL & 6.45 & 5.04 & 5.79 & $-0.67^{\ddagger}(-0.77,-0.57)$ & $+0.75^{\ddagger}(+0.61,+0.89)$ \\
\hline
\end{tabular}

Notes: *4I I patients had follow-up information and the follow-up lipid values of the remaining 653 patients were imputed from their initial lipid values (baseline at the time of

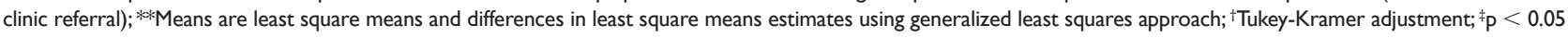
Abbreviations: CRRC, Cardiovascular Risk Reduction Clinic; HDL-C, high-density lipoprotein-cholesterol; LDL-C, low-density lipoprotein-cholesterol;TC, total cholesterol; TG, triglycerides. 
lipid-lowering therapy medication recommendations and adjustments, the significant changes in the lipid profiles observed in the clinic might also be attributed to the structured, one-to-one education and counseling that patients received with the CRRC multidisciplinary team for dietary modification, alcohol consumption, exercise, and smoking cessation during clinic follow-up.

As the primary outcome, we assessed the effectiveness of the CRRC in managing dyslipidemias in 1,064 patients over a 10-year period, demonstrating statistically significant improvements in all lipid parameters. These improvements were achieved over a median of 2.41 years following last clinic follow-up. We successfully obtained the recent lipid profiles, measured $\geq 6$ months after discharge from the CRRC, from referring primary care physicians for $39 \%$ of the eligible patients. The reasons for follow-up lipid profile results not being obtained in the other 653 patients were provided. Interestingly, there were 83 patients $(7.8 \%)$ still under the referring physician's care in whom no recent follow-up lipid screen had been ordered. The lack of follow-up lipid screening is surprising, since it is standard practice for the clinic physicians to recommend the referring physician continue to assess the patients' lipid profile every 6 to 12 months after discharge from the clinic. However, given the patient volume and busy clinical practices of most primary care physicians, some may have delayed ordering follow-up up lipid panels for longer periods of time because they or their patients had the general impression that the lipid profiles were already optimized.

The worst-case analysis, in which the missing follow-up lipid values for 653 patients were imputed, demonstrated that approximately $50 \%$ of the improvement in the lipid parameters was lost between discharge and follow-up for the entire study population of 1064 patients. This observation is not surprising since we imputed the original baseline values for 653 patients (61.4\%) in this analysis; however, one could argue that we should have expected to have observed a greater loss in the benefit in the mean lipid levels in this cohort following discharge, consistent with the proportion of patients for whom the baseline lipid values were imputed. This analysis demonstrated that overall change in each lipid parameter between the initial clinic visit and follow-up remained both statistically and clinically significant.

Based on data from a meta-analysis by Baigent and colleagues (2005), the $0.97 \mathrm{mmol} / \mathrm{L}$ mean reduction in LDL-C that was observed in the 411 patients in whom follow-up lipid profiles were obtained (Table 5) would be expected to result in an $18.4 \%$ reduction in CHD mortality and a $11.6 \%$ reduction in all-cause mortality after 5 years of treatment; however, based on our worst-case analysis (Table 6), the $0.39 \mathrm{mmol} / \mathrm{L}$ would be expected to reduce CHD mortality by $7.4 \%$ reduce all-cause mortality $4.7 \%$ after 5 years of treatment. Undeniably, these statistically significant changes in lipid parameters should translate into clinically significant improvements in patient outcomes over time.

No attempt was made in this study to determine the percentage of patients who successfully achieved their cholesterol goals given that there were no published guidelines for lipid targets during the earlier years of the clinic and guideline recommendations at various times over the 10-year study period were different. This was a retrospective evaluation; therefore, this study is subject to the same limitations as any retrospective study. However, the CRRC has been consistently staffed by a relatively small number of physicians who followed the same general charting procedures during the study period. In addition, the investigators utilized both inpatient and outpatient clinic charts to obtain the patient data which broadened the source of reliable documentation. We were unable to determine if there were other variables in addition to attending the CRRC, which may have influenced the sustained benefits that were observed in the lipid profiles of follow-up patients. Given the consistency in all lipid parameters between the final in-clinic results and the most recent profiles following CRRC discharge, it seems logical to assume that both patients and referring physicians adhered to the recommendations implemented in the clinic; however, we cannot definitively state that there were not other factors that contributed to this sustained benefit. While it was beyond the scope of this study to determine medication and lifestyle modification adherence among patients, this information would be helpful in determining how much of the observed long-term lipid improvements were the result of the CRRC management efforts. Finally, this study was conducted in a single clinic with its own unique patient referral biases, specific clinic experiences, and multidisciplinary staffing pattern which may potentially limit the generalizability of these findings to other specialty clinic practices. The findings of our study may only be applicable to other multidisciplinary clinics, which utilize a similar multifactorial risk-reduction model.

The results of this study suggest that a formally structured CRRC has a significant positive impact on improving important lipid parameters. These improvements appear to be sustained over the long-term after patients are discharged from the CRRC. This evidence lends support to the belief that patients can be discharged from these clinics once they are optimally managed, without compromising the improvements achieved in their CV risk profile. Given that 
the goal of specialty lipid clinics is to prevent cardiac events in patients with CHD or at high-risk for developing CVD in the future, discharging patients once they have their $\mathrm{CV}$ risks optimally managed will facilitate a greater number of patients being seen and benefiting from the multidisciplinary, structured approach to risk reduction provided by such clinics.

\section{Acknowledgments}

The authors thank the following staff from the EPICORE (Epidemiology Coordinating and Research) Centre, Division of Cardiology, University of Alberta for their assistance and support with this study: Marilou Hervas-Malo, MSc, Sandra Blitz, MSc, Ruth Dupuit, BSc, Paula Priest, and Bonnie Woloschuk, BSN.

\section{Disclosure}

The University of Alberta CRRC was supported during the period of the study in part by unrestricted grants from Merck Frosst, Parke Davis/Pfizer, and Bristol-Myers Squibb, who had no influence on the conduct of this study. Dr. Majumdar is a Population Health Investigator of the Alberta Heritage Foundation for Medical Research and a New Investigator of the Canadian Institutes of Health Research. Dr. Tsuyuki is supported by the Merck Frosst Chair in Patient Health Management at the University of Alberta. Mr. Damani was supported by an Apotex/PACE pharmacy undergraduate studentship grant from the Association of Faculties of Pharmacy of Canada. Dr. Francis is a Senior Scholar of the Alberta Heritage Foundation for Medical Research.

\section{References}

Anderson KM, Castelli WP, Levy D. 1987. Cholesterol and mortality: 30 years of follow up from the Framingham study. JAMA, 257:2176-80.

Baigent C, Keech A, Kearney PM, et al. 2005. Efficacy and safety of cholesterol-lowering treatment: prospective meta-analysis of data from 90,056 participants in 14 randomized trials of statins. Lancet, 366:1267-78.

Castelli WP, Garrison RJ, Wilson PWF, et al. 1986. Incidence of coronary heart disease and lipoprotein cholesterol levels. JAMA, 256:2835-8.

Cooke CE, Hammerash WJ Jr. 2006. Retrospective review of sex differences in the management of dyslipidemia in coronary heart disease: an analysis of patient data from a Maryland-based health maintenance organization. Clin Ther, 28:591-9.

Fodor JG, Frolich JJ, Genest Jr. JJG, et al. 2000. Recommendations for the management and treatment of dyslipidemia. CMAJ, 162:1441-7.

Garcia Ruiz FJ, Marin Ibanez A, Perez-Jiminez F, et al. 2004. Current lipid management and low cholesterol goal attainment in common daily practice in Spain. The REALITY Study. Pharmacoeconomics, 22(Suppl 3):1-12.

Gavish D, Leibovitz E, Elly I, et al. 2002. Follow-up in a lipid clinic improves the management of risk factors in cardiovascular disease patients. Isr Med Assoc J, 4:694-7.

Grundy SM, Pasternak R, Greenland P, et al. 1999. Assessment of cardiovascular risk by use of multiple-risk-factor assessment equations: a statement for healthcare professionals from the American Heart Association and the American College of Cardiology. Circulation, 100:1481-92.
Harris DE, Gipson GW, Pearson TA, et al. 1998. Lipid lowering in a multidisciplinary clinic compared with primary physician management. Am J Cardiol, 81:929-33.

Heart and Stroke Foundation of Canada. 1999. The changing face of heart disease and stroke in Canada 2000. Laboratory Centre for Disease Control, Health Canada, Statistics Canada, Canadian Institute for Health Information, Canadian Cardiovascular Society, Canadian Stroke Society, Heart Stroke Foundation of Canada; Ottawa, Canada [online]. Accessed on March 15, 2008. URL: http://www.hc-sc.gc.ca/hpb/lcdc/ bcrdd/hdsc2000/pdf/card2ke.pdf.

Koren MJ, Hunninghake DB. 2004. Clinical outcomes in managed-care patients with coronary heart disease treated aggressively in lipidlowering disease management clinics. J Am Coll Card, 44:1772-9.

Majumdar SR, Gurwitz JH, Soumerai SB. 1999. Undertreatment of hyperlipidemia in the secondary prevention of coronary artery disease. J Gen Intern Med, 14:711-7.

McPherson R, Frohlich J, Fodor J, et al. 2006. Canadian Cardiovascular Society position statement - recommendations for the diagnosis and treatment of dyslipidemia and prevention of cardiovascular disease. Can J Cardiol, 22:913-27.

Murray CJ, Lopez AD. 1997. Global mortality, disability, and the contribution of risk factors: Global Burden of Disease Study. Lancet, 349:1436-42.

[NCEP ATP III] National Cholesterol Education Program Expert Panel on Detection, Evaluation, and Treatment of High Blood Cholesterol in Adults (Adult Treatment Panel III). 2002. Third report of the National Cholesterol Education Program (NCEP) Expert Panel on Detection, Evaluation, and Treatment of High Blood Cholesterol in Adults (Adult Treatment Panel III) final report. Circulation, 106:3143-421.

Neaton JD, Blackburn H, Jacobs D, et al. 1992. Serum cholesterol level and mortality findings for men screened in the Multiple Risk Factor Intervention Trial (MRFIT). Arch Intern Med, 152:1490-500.

Olson KL, Rasmussen J, Sandhoff BG, et al. 2005. Lipid management in patients with coronary artery disease by a clinical pharmacy service in a group model health maintenance organization. Arch Intern Med, 165:49-54.

Pearson TA, Laurora I, Chu H, et al. 2000. The lipid treatment assessment project (L-TAP): a multicenter survey to evaluate the percentages of dyslipidemic patients receiving lipid-lowering therapy and achieving low-density lipoprotein cholesterol goals. Arch Intern Med, 160:459-67.

Salant P, Dillman D. 1994. How to conduct your own survey. New York: John Wiley and Sons.

Schrott HG, Bittner V, Vittinghoff E, et al. 1997. Adherence to National Cholesterol Education Program treatment goals in postmenopausal women with heart disease. The Heart Estrogen/Progestin Replacement Study (HERS). The HERS Research Group. JAMA, 277:1281-6.

Shaffer J, Wexler LF. 1995. Reducing low-density lipoprotein cholesterol levels in an ambulatory care system. Arch Intern Med, 155:2330-5.

Stacy TA, Egger A. 2006. Results of a retrospective chart review to determine improvement in lipid goal attainment in patients treated by high-volume prescribers of lipid-modifying drugs. J Manag Care Pharm, 12:745-51.

Stamler J, Wentworth D, Neaton J. 1996. Is relationship between serum cholesterol and risk of premature death from coronary heart disease continuous and graded? Findings in 356,222 primary screenees of the Multiple Risk Factor Intervention Trial (MRFIT). JAMA, 256:2823-7.

Taher TH, Dzavik V, Reteff EM, et al. 2002. Tolerability of statin-fibrate and statin-niacin combination therapy in dyslipidemic patients at high risk for cardiovascular events. Am J Cardiol, 89:390-4.

Wilson TW, Quest DW, Wilson M, et al. 1999. A cardiovascular risk factor reduction clinic. Can J Cardiol, 15:887-91.

Yates S, Annis L, Pippins J, et al. 2001. Does a lipid clinic increase compliance with National Cholesterol Education Program treatment guidelines? Report of a case-matched control study. South Med J, 94:907-9. 
